This item was submitted to Loughborough's Research Repository by the author.

Items in Figshare are protected by copyright, with all rights reserved, unless otherwise indicated.

\title{
Rigorous numerical approximation of escape rates
}

PLEASE CITE THE PUBLISHED VERSION

https://doi.org/10.1088/0951-7715/19/11/002

\section{PUBLISHER}

(C) IOP and London Mathematical Society

\section{VERSION}

AM (Accepted Manuscript)

\section{PUBLISHER STATEMENT}

This work is made available according to the conditions of the Creative Commons Attribution-NonCommercialNoDerivatives 4.0 International (CC BY-NC-ND 4.0) licence. Full details of this licence are available at: https://creativecommons.org/licenses/by-nc-nd/4.0/

\section{LICENCE}

CC BY-NC-ND 4.0

\section{REPOSITORY RECORD}

Bahsoun, Wael. 2019. "Rigorous Numerical Approximation of Escape Rates". figshare. https://hdl.handle.net/2134/26319. 


\title{
RIGOROUS NUMERICAL APPROXIMATION OF ESCAPE RATES
}

\author{
WAEL BAHSOUN
}

\begin{abstract}
An interval map with holes is a mathematical model which is used in the study of nonequilibrium statistical mechanics. We use Ulam's method to approximate the escape rate for an interval map with holes and find a bound on the approximation error.
\end{abstract}

\section{INTRODUCTION}

Consider an infinite set of identical particles evolving independently in a box $I$ according to a stochastic process $T$ which throws some of the particles outside of $I$. If a particle escapes from $I$ under the process $T$, it will never come back again. Such systems are of central interest in the study of nonequilibrium statistical mechanics $[6,7,15]$.

A physical quantity associated to this problem is the escape rate of the particles from the box. In [6], a theoretic escape rate formula is found. However, it is often impossible to compute the exact escape rate using this formula.

Interval maps with holes are one possible mathematical model of such systems. In this paper we deal with Lasota-Yorke maps with holes [2, 12]. These maps, which were first introduced in [14], are defined as follows:

Let $T: \mathbb{R} \rightarrow \mathbb{R}$ be a piecewise monotonic and piecewise $C^{2}$ nonsingular transformation (with respect to Lebesgue measure) and piecewise expanding. Let $H$ be a finite union of disjoint open intervals, $H=\bigcup_{j=1}^{L} H_{j}, H \subset[0,1], T^{n}(H) \cap[0,1]=\emptyset$ for all $n \in \mathbb{N}$, and $T([0,1] \backslash H)=[0,1]$. Define $T_{H}:[0,1] \backslash H \rightarrow[0,1]$ to be the restriction of $T$ to $[0,1] \backslash H$.

This model was studied in [2] where existence of an absolutely continuous conditionally invariant measure (see Definition 3.1) was proved under certain conditions. Our goal is to present a numerical technique to approximate the escape rate for an interval map with holes and find a bound on the approximation error. This technique is based on Ulam's scheme [16] and it relies on the abstract perturbation result of [9] and its numerical counterpart [11].

In Section 2 we present the abstract stability theorem of [9]. In fact, we need the statement of the this result since the constants involved in it are needed in

Date: January 21, 2009.

1991 Mathematics Subject Classification. Primary 37A05, 37E05.

Key words and phrases. Frobenius-Perron operator, Interval Maps, Escape Rates.

W.B. is a recipient of PIMS postdoctoral fellowship. 
our numerical computations. Section 3 is devoted to numerical approximation of the escape rate. In particular, we prove, in Lemma 3.10, a result which enables us to use the computer and estimate the $B V$ norm of the resolvent of a finite rank operator. Our main result in this section is obtaining an algorithm which can be implemented to compute the approximate escape rate. In Section 4, we obtain rates of convergence of the approximation scheme of Section 3. Our main result in this section is Theorem 4.6. In Section 5, we show that the algorithm of Section 3 is actually feasible to implement. In particular, we use the algorithm to give rigorous approximation of the escape rate of a certain Lasota-Yorke map with holes.

\section{Abstract perturbation Result}

Let $(I, \mathfrak{B}, \lambda)$ be the measure space where $I=[0,1], \mathfrak{B}$ is the Borel $\sigma$-algebra and $\lambda$ is Lebesgue measure. Let $L^{1}=L^{1}(I, \mathfrak{B}, \lambda)$. For $f \in L^{1}$, we define

$$
V f=\inf _{\bar{f}}\{\operatorname{var} \bar{f}: f=\bar{f} \text { a.e. }\} \text {, }
$$

where

$$
\operatorname{var} \bar{f}=\sup \left\{\sum_{i=0}^{l-1}\left|\bar{f}\left(x_{i+1}\right)-\bar{f}\left(x_{i}\right)\right|: 0=x_{0}<x_{1}<\cdots<x_{l}=1\right\} .
$$

We denote by $B V$ the space of functions of bounded variation on $I$ equipped with the norm $\|\cdot\|_{B V}=V(\cdot)+\|\cdot\|_{1}[5]$.

Let $P_{i}: B V(I) \rightarrow B V(I)$ be two bounded linear operators, i=1,2. We assume that: For $f \in L^{1}$

$$
\left\|P_{i} f\right\|_{1} \leq\|f\|_{1}
$$

and $\exists \alpha \in(0,1), A>0$ and $B \geq 0$ such that

$$
\left\|P_{i}^{n} f\right\|_{B V} \leq A \alpha^{n}\|f\|_{B V}+B\|f\|_{1} \quad \forall n \in \mathbb{N} \forall f \in B V(I) .
$$

Further, we introduce the mixed operator norm:

$$
\left\|P_{i}\right\|\left\|=\sup _{\|f\|_{B V} \leq 1}\right\| P_{i} f \|_{1}
$$

For any bounded linear operator $P: B V \rightarrow B V$ with spectrum $\sigma(P)$, consider the set

$$
V_{\delta, r}(P)=\{z \in \mathbb{C}:|z| \leq r \text { or } \operatorname{dist}(z, \sigma(P)) \leq \delta\}
$$

Since the complement of $V_{\delta, r}(P)$ belongs to the resolvent of $P$, it follows that ([5] Lemma 11, VII.6.10)

$$
H_{\delta, r}(P)=\sup _{z}\left\{\left\|(z-P)^{-1}\right\|_{B V}: z \in \mathbb{C} \backslash V_{\delta, r}\right\}<\infty .
$$

Remark 2.1. $\alpha$ in (2.2) is an upper bound on the essential spectral radius of $P_{i}[1]$.

Theorem 2.2. $[9,11]$ Consider two operators $P_{i}: B V(I) \rightarrow B V(I)$ which satisfy (2.1) and (2.2). For $r \in(\alpha, 1)$, let

$$
\begin{gathered}
n_{1}=\left\lceil\frac{\ln 2 A}{\ln r / \alpha}\right\rceil \\
C=r^{-n_{1}} ; \quad D=A(A+B+2) \\
n_{2}=\left\lceil\frac{\ln 8 B D C H_{\delta, r}\left(P_{1}\right)}{\ln r / \alpha}\right\rceil .
\end{gathered}
$$


If

$$
\left\|\left|P_{1}-P_{2} \|\right| \leq \frac{r^{n_{1}+n_{2}}}{8 B\left(H_{\delta, r}\left(P_{1}\right) B+(1-r)^{-1}\right)} \stackrel{\text { def }}{=} \varepsilon_{1}\left(P_{1}, r, \delta\right)\right.
$$

then for each $z \in \mathbb{C} \backslash V_{\delta, r}\left(P_{1}\right)$, we have

$$
\left\|\left(z-P_{2}\right)^{-1} f\right\|_{B V} \leq \frac{4(A+B)}{1-r} r^{-n_{1}}\|f\|_{B V}+\frac{1}{2 \varepsilon_{1}}\|f\|_{1} .
$$

Set

$$
\begin{gathered}
\gamma=\frac{\ln (r / \alpha)}{\ln (1 / \alpha)}, \\
a=\frac{8\left[2 A(A+B)+(1-r)^{-1}\right](A+B)^{2} r^{-n_{1}}+1}{1-r}
\end{gathered}
$$

and

$$
b=2\left[\left(4(A+B)^{2}(D+B)+B\right)(1-r)^{-1} r^{-n_{1}}+B\right] .
$$

If

$$
\begin{aligned}
\left\||| P_{1}-P_{2}\right\| \mid & \leq \min \left\{\varepsilon_{1}\left(P_{1}, r, \delta\right),\left[\frac{r^{n_{1}}}{4 B\left(H_{\delta, r}\left(P_{1}\right)(D+B)+2 A(A+B)+(1-r)^{-1}\right)}\right]^{\gamma}\right\} \\
& \stackrel{\text { def }}{=} \varepsilon_{0}\left(P_{1}, r, \delta\right)
\end{aligned}
$$

then for each $z \in \mathbb{C} \backslash V_{\delta, r}\left(P_{1}\right)$, we have

||$\left|\left(z-P_{2}\right)^{-1}-\left(z-P_{1}\right)^{-1}\right||| \leq||\left|P_{1}-P_{2}\right|||^{\gamma}\left(a\left\|\left(z-P_{1}\right)^{-1}\right\|_{B V}+b\left\|\left(z-P_{1}\right)^{-1}\right\|_{B V}^{2}\right)$.

Corollary 2.3. [9, 11] If $\left\|\left|P_{1}-P_{2} \|\right| \leq \varepsilon_{1}\left(P_{1}, r, \delta\right)\right.$ then $\sigma\left(P_{2}\right) \subset V_{\delta, r}\left(P_{1}\right)$. In addition, if $\left\|\left|P_{1}-P_{2} \|\right| \leq \varepsilon_{0}\left(P_{1}, r, \delta\right)\right.$, then in each connected component of $V_{\delta, r}\left(P_{1}\right)$ that does not contain 0 both $\sigma\left(P_{1}\right)$ and $\sigma\left(P_{2}\right)$ have the same multiplicity; i.e., the associated spectral projections have the same rank.

\section{ULAM'S METHOD FOR ESCAPE RATES}

Let $T$ be an interval map with holes and denote by $P$ the Perron-Frobenius operator associated with $T$ [1]. Let $f \geq 0, \int_{I} f d \lambda=1$ and denote by $\operatorname{supp}(f)$ the support of $f$. Suppose for a moment that a.e. $x$ eventually escapes from $I$, then for some $n \geq 1, \lambda\left(T^{-n}(I)\right)<\lambda(\operatorname{supp}(f))$ and we have

$$
\left\|P^{n} f\right\|_{1}=\int_{I} P^{n} f d \lambda=\int_{T^{-n_{I}}} f d \lambda<\int_{I} f d \lambda=\|f\|_{1} .
$$

Thus, $P$ eventually decreases the norm of functions. Consequently $T$ cannot preserve a measure which is absolutely continuous with respect to Lebesgue measure. In this case, the measure which describes the dynamics is given by the following definition.

Definition 3.1. A probability measure $\mu$ on $[0,1], d \mu=g d \lambda$, is said to be a $T$ absolutely continuous conditionally invariant measure if there exists $0<\rho<1$ such that $P g=\rho g$. 
For results on the existence of absolutely continuous conditionally invariant measures we refer the reader to $[2,3,12]$ and to the recent survey article [4].

The escape rate of the points through the holes under the iteration of $T$ is defined by $-\ln \rho$ where $\rho$ is the dominant eigenvalue of the Perron-Frobenius operator associated with $T$. In this section, we present a numerical method for estimating $\rho$. We assume:

(A1) $\exists \alpha \in(0,1)$, and $B_{0} \geq 0$ such that $\forall f \in B V(I)$

$$
V P f \leq \alpha V f+B_{0}\|f\|_{1}
$$

(A2) $P$, as operator on $B V(I)$, has a dominant eigenvalue $\rho$ such that:

$$
\alpha<\rho<1 \text {. }
$$

Remark 3.2. Since $\alpha$ is an upper bound on the essential spectral radius of $P$, any eigenvalue of $P$ with modulus bigger than $\alpha$ is an isolated eigenvalue of finite multiplicity. Thus, $\exists r_{0}>\alpha$ and $\delta_{0}>0$ such that any eigenvalue $\rho_{i}$ of $P$, with $\left|\rho_{i}\right|>r_{0}$, satisfies:

(1) $B\left(\rho_{i}, 2 \delta_{0}\right) \cap B\left(0, r_{0}\right)=\emptyset$;

(2) $B\left(\rho_{i}, 2 \delta_{0}\right) \cap B\left(\rho_{j}, 2 \delta_{0}\right)=\emptyset$ for $i \neq j$.

Remark 3.3. Given a Lasota-Yorke map with holes, $\alpha$ and $B_{0}$ in the inequality of (A1) are computable.

We now present Ulam's approximation of $P$. Let $\eta$ be a finite partition of $I$ into intervals. Let $\operatorname{mesh}(\eta)$ be the mesh size of $\eta$; i.e, the maximum length of an interval in $\eta$, and let $\mathfrak{B}_{\eta}$ be the finite $\sigma$-algebra associated with $\eta$. For $f \in L^{1}$, let

$$
\Pi_{\eta} f=\mathbb{E}\left(f \mid \mathfrak{B}_{\eta}\right),
$$

where $\mathbb{E}\left(\cdot \mid \mathfrak{B}_{\eta}\right)$ denotes the conditional expectation with respect to $\mathfrak{B}_{\eta}$. Then, if $x \in I_{\eta} \in \eta$

$$
\left(\Pi_{\eta} f\right)(x)=\frac{1}{\lambda\left(I_{\eta}\right)} \int_{I_{\eta}} f d \lambda
$$

Define

$$
P_{\eta}=\Pi_{\eta} \circ P \circ \Pi_{\eta} .
$$

$P_{\eta}$ is called Ulam's approximation of $P$. Using the basis $\left\{\frac{1}{\lambda\left(I_{\eta}\right)} \chi_{I_{\eta}}\right\}$ in $L^{1}, P_{\eta}$ can be represented by a (row) substochastic matrix acting on vectors from $\mathbb{R}^{d(\eta)}$ by left multiplication. The entries of Ulam's matrix are given by:

$$
P_{I_{\eta} J_{\eta}}=\frac{\lambda\left(I_{\eta} \cap T^{-1} J_{\eta}\right)}{\lambda\left(I_{\eta}\right)}
$$

Lemma 3.4. $P_{\eta}$ has a positive dominant eigenvalue $\rho_{\eta} \leq 1$.

Proof. By definition $P_{\eta}$ is a non-negative matrix. Therefore, the existence of the positive dominant eigenvalue $\rho_{\eta}$ follows from the Perron-Frobenius Theorem on non-negative matrices [10]. Moreover, for $f \in L^{1}$, we have

$$
\left\|P_{\eta} f\right\|_{1}=\left\|\left(\Pi_{\eta} \circ P \circ \Pi_{\eta}\right) f\right\|_{1} \leq\left\|P \Pi_{\eta} f\right\|_{1} \leq\left\|\Pi_{\eta} f\right\|_{1} \leq\|f\|_{1} .
$$

Thus, $\rho_{\eta} \leq 1$. 
The following lemma mainly states that the projection operator $\Pi_{\eta}$ does not increase variation when $\eta$ is defined as above. This is a well known fact [13]. We will denote the identity operator by $\mathbf{1}$.

Lemma 3.5. For $f \in B V(I)$ and $\varepsilon=\operatorname{mesh}(\eta)$ we have

(1) $V\left(\Pi_{\eta} f\right) \leq V(f)$;

(2) $\left\|f-\Pi_{\eta} f\right\|_{1} \leq \varepsilon V(f)$.

\section{Lemma 3.6.}

(1) $P_{\eta}$ and $P$ satisfy (2.2) with the same constants;

(2) $\left\||| P_{\eta}-P\right\| \mid \leq \Gamma \varepsilon$, where $\Gamma=\max \left\{\alpha+1, B_{0}\right\}$ and $\varepsilon=\operatorname{mesh}(\eta)$.

Proof. Firstly, for $f \in B V(I)$, by (A1), for any $n \in \mathbb{N}$

$$
V P^{n} f \leq \alpha^{n} V f+B_{1}\|f\|_{1},
$$

where $B_{1}=\frac{B_{0}}{1-\alpha}$. Thus, $P$ satisfies (2.2) with $\mathrm{A}=1$, and $B=B_{1}+1$. By the first statement of Lemma 3.5, we have

$$
V P_{\eta} f=V\left(\left(\Pi_{\eta} \circ P \circ \Pi_{\eta}\right) f\right) \leq V\left(P \Pi_{\eta} f\right) \leq \alpha V f+B_{0}\|f\|_{1} .
$$

Thus $P_{\eta}$ satisfies (2.2) with $\mathrm{A}=1$, and $B=B_{1}+1$. This completes the proof of the first part of the lemma. By the second statement of Lemma 3.5 we obtain

$$
\begin{aligned}
\left\|\left(P_{\eta}-P\right) f\right\|_{1} & \leq\left\|\left(\Pi_{\eta} \circ P \circ \Pi_{\eta}-P \circ \Pi_{\eta}\right) f\right\|_{1}+\left\|\left(P \circ \Pi_{\eta}-P\right) f\right\|_{1} \\
& =\left\|\left(\Pi_{\eta}-\mathbf{1}\right) P \Pi_{\eta} f\right\|_{1}+\left\|P\left(\Pi_{\eta}-\mathbf{1}\right) f\right\|_{1} \\
& \leq \varepsilon V\left(P \Pi_{\eta} f\right)+\varepsilon V f \\
& \leq \varepsilon\left((\alpha+1) V f+B_{0}\|f\|_{1}\right) \\
& \leq \varepsilon \Gamma\|f\|_{B V} .
\end{aligned}
$$

Remark 3.7. Our goal is to approximate the dominant eigenvalue of $P$ by the dominant eigenvalue of $P_{\eta}$ for a suitable $\eta$. However, the constants in Theorem 2.2 depend on the norm of the resolvent of the operator. Since all these constants are computable for $P_{\eta}$ (we will show this later in this section), we use Theorem 2.2 with $P_{\eta}$ as the first operator and $P$ as the second operator.

First, we need the following lemma to show that we can really use Theorem 2.2 with $P_{\eta}$ as the first operator and $P$ as the second operator. The proof is based on the proof of Lemma 4.2 of [11].

Lemma 3.8. [11] Given $P, \delta>0$ and $r>\alpha$, there exists $\varepsilon_{2}>0$ such that for each $\eta$ with $0<\operatorname{mesh}(\eta) \leq \varepsilon_{2}$, we have

$$
\operatorname{mesh}(\eta) \leq(2 \Gamma)^{-1} \varepsilon_{0}\left(P_{\eta}, r, \delta\right),
$$

and

$$
\left\|P_{\eta}-P\right\| \mid \leq \frac{1}{2} \varepsilon_{0}\left(P_{\eta}, r, \delta\right) .
$$

Proof. Since (3.2) follows from (3.1) by Lemma 3.6, we only prove (3.1). Let $P, P_{\eta}$ be $P_{1}, P_{2}$ of Theorem 2.2 respectively and $\varepsilon=\operatorname{mesh}(\eta)$. There exist a $\delta_{0}$ and an $r_{0}, 0<\delta_{0} \leq \delta$ and $\alpha<r_{0} \leq r$, such that for any eigenvalue $\rho_{i}$ of $P$, with $\left|\rho_{i}\right|>\alpha$, we have: 
(1) $B\left(\rho_{i}, 2 \delta_{0}\right) \cap B\left(0, r_{0}\right)=\emptyset$;

(2) $B\left(\rho_{i}, 2 \delta_{0}\right) \cap B\left(\rho_{j}, 2 \delta_{0}\right)=\emptyset$ for $i \neq j$.

If $2 \Gamma \varepsilon \leq \varepsilon_{0}\left(P, r_{0}, \delta_{0} / 2\right)\left(\varepsilon_{0} \leq \varepsilon_{1}\left(P, r_{0}, \delta_{0} / 2\right)\right)$, then there exists a constant $c_{*}$ such that

$$
\sup _{z \in \mathbb{C} \backslash V_{\delta_{0} / 2, r_{0}}(P)}\left\|\left(z-P_{\eta}\right)^{-1}\right\|_{B V} \leq c_{*} .
$$

In particular, $\sigma\left(P_{\eta}\right) \subset V_{\delta_{0} / 2, r_{0}}(P)$. Let $\rho \in \sigma(P)$ with $|\rho|>r_{0}$. Then, by Corollary 2.3 , there exists a $\rho_{\eta} \in \sigma\left(P_{\eta}\right)$ such that $\left|\rho-\rho_{\eta}\right| \leq \delta_{0} / 2$. Thus, if $\left|z-\rho_{\eta}\right| \geq \delta_{0}$ we obtain

$$
|z-\rho| \geq\left|\rho_{\eta}-z\right|-\left|\rho-\rho_{\eta}\right| \geq \delta_{0} / 2 .
$$

Thus, $V_{\delta_{0} / 2, r_{0}}(P) \subset V_{\delta_{0}, r_{0}}\left(P_{\eta}\right) \subseteq V_{\delta, r}\left(P_{\eta}\right)$; i.e.,

$$
\mathbb{C} \backslash V_{\delta, r}\left(P_{\eta}\right) \subseteq \mathbb{C} \backslash V_{\delta_{0} / 2, r_{0}}(P) .
$$

Therefore,

$$
\sup _{z \in \mathbb{C} \backslash V_{\delta, r}\left(P_{\eta}\right)}\left\|\left(z-P_{\eta}\right)^{-1}\right\|_{B V} \leq \sup _{z \in \mathbb{C} \backslash V_{\delta_{0} / 2, r_{0}}(P)}\left\|\left(z-P_{\eta}\right)^{-1}\right\|_{B V} \leq c_{*} .
$$

Thus, $H_{\delta, r}\left(P_{\eta}\right) \leq c_{*}$ and for fixed $r$ and $\delta, \varepsilon_{0}\left(P_{\eta}, r, \delta\right)$ is uniformly bounded from below. Set

$$
\varepsilon_{2}=\inf \left\{(2 \Gamma)^{-1} \varepsilon_{0}\left(P_{\eta}, r, \delta\right): \varepsilon=\operatorname{mesh}(\eta) \leq(2 \Gamma)^{-1} \varepsilon_{0}\left(P, r_{0}, \delta_{0} / 2\right)\right\}>0 .
$$

Remark 3.9. The main difficulty in applying the above lemma is the computation of $c_{*}$. In fact $\left(z-P_{\eta}\right)^{-1}$ acts on the whole of $B V$ and consequently, its norm cannot be found using the computer. In the next lemma, we show how we deal with this difficulty.

Lemma 3.10. For $f \in B V(I)$ with $\|f\|_{B V}=1$ and $z \in \mathbb{C} \backslash V_{\delta, r}\left(P_{\eta}\right)$ we have:

(1) $\left\|\left(z-P_{\eta}\right)^{-1} f\right\|_{B V} \leq\left(\frac{B_{0}}{r-\alpha}+1\right)\left\|\left(z-P_{\eta}\right)^{-1} \Pi_{\eta} f\right\|_{1}+\frac{1}{r-\alpha}+\frac{3}{r}$;

(2) $\left\|\left(z-P_{\eta}\right)^{-1} \Pi_{\eta} f\right\|_{1} \leq\left\|\left(z-P_{\eta}\right)^{-1} f\right\|_{B V}+\frac{3}{r}$.

Proof. Let $f \in B V(I),\|f\|_{B V}=1$. Firstly, using the fact $P_{\eta}^{i} f=P_{\eta}^{i} \Pi_{\eta} f$ for $i \geq 1$ and the identity

$$
\left(z-P_{\eta}\right)^{-1}=z^{-1}\left(z-P_{\eta}\right)^{-1} P_{\eta}+z^{-1} \cdot \mathbf{1},
$$

we have

$$
\begin{aligned}
\left\|\left(z-P_{\eta}\right)^{-1}\left(f-\Pi_{\eta} f\right)\right\|_{B V} & =\left\|\left(z^{-1}\left(z-P_{\eta}\right)^{-1} P_{\eta}+z^{-1} \cdot \mathbf{1}\right)\left(f-\Pi_{\eta} f\right)\right\|_{B V} \\
& =\left\|\frac{f}{z}-\frac{\Pi_{\eta} f}{z}\right\|_{B V} \leq \frac{1}{|z|}\left\|\left(\Pi_{\eta}-\mathbf{1}\right) f\right\|_{B V} \leq \frac{3}{r} .
\end{aligned}
$$

Now, write $\left(z-P_{\eta}\right)^{-1} \Pi_{\eta} f=h$, then $h=\frac{1}{z}\left(P_{\eta} h+\Pi_{\eta} f\right)$. Therefore, we have

$$
\begin{aligned}
V h & \leq \frac{1}{|z|}\left(V P_{\eta} h+1\right) \\
& \leq \frac{1}{r}\left(\alpha V h+B_{0}\|h\|_{1}+1\right) .
\end{aligned}
$$

By (3.5), we obtain

$$
V h \leq \frac{B_{0}\|h\|_{1}+1}{r-\alpha}
$$


and consequently,

$$
\left\|\left(z-P_{\eta}\right)^{-1} \Pi_{\eta} f\right\|_{B V} \leq\left(\frac{B_{0}}{r-\alpha}+1\right)\left\|\left(z-P_{\eta}\right)^{-1} \Pi_{\eta} f\right\|_{1}+\frac{1}{r-\alpha} .
$$

By (3.4) and (3.6) the first part of the lemma follows. For the second part, by (3.4), we have

$$
\begin{aligned}
\left\|\left(z-P_{\eta}\right)^{-1} \Pi_{\eta} f\right\|_{1} & \leq\left\|\left(z-P_{\eta}\right)^{-1} \Pi_{\eta} f\right\|_{B V} \\
& \leq\left\|\left(z-P_{\eta}\right)^{-1} f\right\|_{B V}+\left\|\left(z-P_{\eta}\right)^{-1}\left(\Pi_{\eta} f-f\right)\right\|_{B V} \\
& \leq\left\|\left(z-P_{\eta}\right)^{-1} f\right\|_{B V}+\frac{3}{r} .
\end{aligned}
$$

Remark 3.11. One can use the matrix representation of $P_{\eta}$ and the computer to find $\left\|\left(z-P_{\eta}\right)^{-1} \Pi_{\eta} f\right\|_{1}$ since $\Pi_{\eta} f$ is just a vector.

Let

$H_{\delta, r}^{*}\left(P_{\eta}\right) \stackrel{\text { def }}{=} \sup \left\{\left(\frac{B_{0}}{r-\alpha}+1\right)\left\|\left(z-P_{\eta}\right)^{-1} \Pi_{\eta} f\right\|_{1}+\frac{1}{r-\alpha}+\frac{3}{r}:\|f\|_{B V}=1, z \in \mathbb{C} \backslash V_{\delta, r}\left(P_{\eta}\right)\right\}$,

and

$$
\begin{aligned}
\varepsilon_{0}^{*}\left(P_{\eta}, \delta, r\right) & =\min \left\{\frac{r^{n_{1}+\left\lceil\frac{\ln 8 B D C H_{\delta, r}^{*}\left(P_{\eta}\right)}{\ln r / \alpha}\right\rceil}}{8 B\left(H_{\delta, r}^{*}\left(P_{\eta}\right) B+(1-r)^{-1}\right)},\right. \\
& {\left.\left[\frac{r^{n_{1}}}{4 B\left(H_{\delta, r}^{*}\left(P_{\eta}\right)(D+B)+2(1+B)+(1-r)^{-1}\right)}\right]^{\gamma}\right\} . }
\end{aligned}
$$

\section{Lemma 3.12.}

(1) $\varepsilon_{0}^{*}\left(P_{\eta}, \delta, r\right)$ is uniformly bounded below;

(2) $\varepsilon_{0}^{*}\left(P_{\eta}, \delta, r\right) \leq \varepsilon_{0}\left(P_{\eta}, \delta, r\right)$;

(3) $\operatorname{mesh}(\eta) \leq(2 \Gamma)^{-1} \varepsilon_{0}^{*}\left(P_{\eta}, \delta, r\right)$ implies mesh( $\left.\eta\right)$ satisfies (3.1).

Proof. By the proof of Lemma 3.8, $\varepsilon_{0}\left(P_{\eta}, \delta, r\right)$ is uniformly bounded below by a positive number. Then using part 2 of Lemma 3.10 , we see that $\varepsilon_{0}^{*}\left(P_{\eta}, \delta, r\right)$ is uniformly bounded below by a positive number. The second part follows from part (1) of lemma 3.10. The last part of the lemma is a consequence of the second part.

Based on the fact that $\varepsilon_{0}^{*}\left(P_{\eta}, \delta, r\right)$ is really computable, we present an algorithm which can be implemented to approximate $\rho$ with a specified tolerance, $\tau$, on the approximation error. This could not have been achieved using $\varepsilon_{0}\left(P_{\eta}, \delta, r\right)$.

\section{Algorithm I}

(1) Given an interval map with holes whose $P$ satisfies conditions (A1) and (A2). Then, $\alpha$ and $B_{0}$ are given. Let $\tau>0$ be a given tolerance on the error.

(2) Pick $\delta=\frac{1}{2 k}$ and $r=\alpha+\frac{1}{k}$ for $k \in \mathbb{N}$ such that $\delta \leq \tau$ and $r<1$.

(3) Feed in a partition of $I$ into intervals. Call it $\eta$.

(4) Compute $\varepsilon$ the mesh size of $\eta$. 
(5) Find $P_{\eta}=\left(P_{I_{\eta} J_{\eta}}\right)$ where

$$
P_{I_{\eta} J_{\eta}}=\frac{\lambda\left(I_{\eta} \cap T^{-1} J_{\eta}\right)}{\lambda\left(I_{\eta}\right)}
$$

(6) Compute the following: $H_{\delta, r}^{*}\left(P_{\eta}\right), n_{1}=\left\lceil\frac{\ln 2}{\ln r / \alpha}\right\rceil, C=r^{-n_{1}}, D=3+B$, $n_{2}=\left\lceil\frac{\ln 8 B D C H_{\delta, r}^{*}\left(P_{\eta}\right.}{\ln r / \alpha}\right\rceil, \gamma=\frac{\ln (r / \alpha)}{\ln (1 / \alpha)}, B=\frac{B_{0}}{1-r}+1, \Gamma=\max \left\{1+\alpha, B_{0}\right\}$.

(7) Check if $\varepsilon \leq(2 \Gamma)^{-1} \varepsilon_{0}^{*}\left(P_{\eta}, \delta, r\right)$.

If (7) is not satisfied, feed in a new $\eta$ with a smaller mesh size and repeat (3)-(7); otherwise, continue.

(8) List the eigenvalues of $P_{\eta}$ whose modulus is bigger than $r: \rho_{\eta, i}, i=1, \ldots, d$.

(a) $P_{\eta}=Q R$ decomposition, where $Q$ is an orthogonal matrix and $R$ is an upper triangular matrix.

(b) $R Q=Q^{T} P_{\eta} Q$.

(c) $P_{\eta, 1}=P_{\eta}, P_{\eta, k}=Q_{k} R_{k}, P_{\eta, k+1}=Q_{k}^{T} P_{\eta, k} Q_{k}$.

This iterative method is called the QR method [17]. Most mathematical software uses the QR method to compute eigenvalues.

(9) Find $\rho_{\eta}$ the dominant eigenvalue of $P_{\eta}$ and define:

$$
C L=\left\{\text { all the eigenvalues from the list which are in } B\left(\rho_{\eta}, \delta\right)\right\} .
$$

(10) Check:

(a) The list in (8) is not empty.

(b) $\bar{B}\left(\rho_{\eta}, \delta\right) \cap \bar{B}(0, r)=\emptyset$;

(c) If $\rho_{\eta, i} \notin C L$, then $\bar{B}\left(\rho_{\eta, i}, \delta\right) \cap \bar{B}\left(\rho_{\eta}, \delta\right)=\emptyset$.

(11) If (10) is satisfied, report $\left|\rho-\rho_{\eta}\right|<\delta$; otherwise, multiply $k$ by 2 and repeat steps (2)-(11) starting with the last $\eta$ that satisfied (7).

Proposition 3.13. The above algorithm stops after finitely many steps.

Proof. By Lemma 3.12, for each $\delta>0$ and $r>\alpha \exists \varepsilon=\operatorname{mesh}(\eta)>0$ such that

$$
\varepsilon<(2 \Gamma)^{-1} \varepsilon_{0}^{*}\left(P_{\eta}, r, \delta\right) .
$$

Therefore, the internal loop of the algorithm (2)-(7) stops after finitely many steps. We now prove that the outer loop of the algorithm stops after finitely many steps. There exist a $K \in \mathbb{N}, K<+\infty$, such that $\delta=\frac{1}{2 K}<\delta_{0}, r=\alpha+\frac{1}{K}<r_{0}$ and $\eta$ with $\varepsilon=\operatorname{mesh}(\eta)>0$ such that

(1) $\varepsilon<(2 \Gamma)^{-1} \varepsilon_{0}^{*}\left(P_{\eta}, \alpha+\frac{1}{K}, \frac{1}{2 K}\right)$;

(2) $\varepsilon<(2 \Gamma)^{-1} \varepsilon_{0}\left(P, \alpha+\frac{1}{K}, \frac{1}{2 K}\right)$.

(1) implies $\sigma(P) \subset V_{\delta, r}\left(P_{\eta}\right)$ and (2) implies $\sigma\left(P_{\eta}\right) \subset V_{\delta, r}(P) \subset V_{\delta_{0}, r_{0}}(P)$. Thus, $\rho \in B\left(\rho_{\eta}, \delta\right), C L \subset B(\rho, \delta)$, and any $P_{\eta}$ eigenvalue which is not in $C L$ is contained in $V_{\delta_{0}, r_{0}}(P) \backslash B(\rho, \delta)$. By Remark 3.2, (11) of the algorithm is satisfied for this $K$.

\section{Rates of Convergence}

In this section, in addition to assumptions (A1) and (A2), we assume:

(A3) $\rho$, the dominant eigenvalue of $P$, is simple. 
We first present an algorithm which enables us to find a $P_{\eta}$ whose dominant eigenvalue, $\rho_{\eta}$, is simple and within a specified distance from $\rho$.

\section{Algorithm II}

(1) Given an interval map with holes whose $P$ satisfies conditions (A1), (A2) and (A3). Then, $\alpha$ and $B_{0}$ are given. Let $\tau>0$ be a given tolerance on the error.

(2)-(9) are the same as in Algorithm I.

(10) (a) Check that $C L=\left\{\rho_{\eta}\right\}$.

(b) $B\left(\rho_{\eta}, 2 \delta\right) \cap B(0, r)=\emptyset$;

(c) If $\rho_{\eta, i} \notin C L$, then $B\left(\rho_{\eta, i}, 2 \delta\right) \cap B\left(\rho_{\eta}, 2 \delta\right)=\emptyset$.

(11) is the same as in Algorithm I.

Proposition 4.1. Algorithm II stops after finitely many steps.

Proof. The internal loop (2)-(7) of Algorithm II stops after finitely many steps for the same reasons as in Proposition 3.13. We now prove that the outer loop stops after finitely many steps. There exist a $K \in \mathbb{N}, K<+\infty$, such that $\delta=\frac{1}{2 K}<\delta_{0}$, $r=\alpha+\frac{1}{K}<r_{0}$ and $\eta$ with $\varepsilon=\operatorname{mesh}(\eta)>0$ such that

(1) $\varepsilon<(2 \Gamma)^{-1} \varepsilon_{0}^{*}\left(P_{\eta}, \alpha+\frac{1}{K}, \frac{1}{2 K}\right)$;

(2) $\varepsilon<(2 \Gamma)^{-1} \varepsilon_{0}\left(P, \alpha+\frac{1}{K}, \frac{1}{2 K}\right)$.

(1) implies $\sigma(P) \subset V_{\delta, r}\left(P_{\eta}\right)$ and (2) implies $\sigma\left(P_{\eta}\right) \subset V_{\delta, r}(P) \subset V_{\delta_{0}, r_{0}}(P)$. Thus, $\rho \in B\left(\rho_{\eta}, \delta\right)$ and $\rho_{\eta} \in B(\rho, \delta)$. Since $\rho$ is simple, then by Corollary 2.3, $\rho_{\eta}$ is the only eigenvalue of $P_{\eta}$ in $B(\rho, \delta)$ and any non dominant $P_{\eta}$ eigenvalue is contained in $V_{\delta_{0}, r_{0}}(P) \backslash B(\rho, \delta)$. By Remark 3.2, (11) of the algorithm is satisfied for this $K$.

Let $\varepsilon_{k}=\operatorname{mesh}\left(\eta_{k}\right), \delta_{k}$ and $r_{k}$, such that $\varepsilon_{k}<(2 \Gamma)^{-1} \varepsilon_{0}\left(P_{\eta}, \delta_{k}, r_{k}\right)$ and $\left|\rho_{\eta_{k}}-\rho\right|<\delta_{k}$, where $\rho_{\eta_{k}}$ is the dominant eigenvalue of $P_{\eta_{k}}$, be given from Algorithm II.

Lemma 4.2. Let $\varepsilon=\operatorname{mesh}(\eta)<\varepsilon_{k}$, then for $z \in \mathbb{C} \backslash V_{\delta_{k}, r}\left(P_{\eta_{k}}\right)$ we have

$$
\left\|\left(z-P_{\eta}\right)^{-1}\right\|_{B V} \leq\left(\frac{4(A+B)}{1-r_{k}} r_{k}^{n_{1}}+\frac{1}{2 \varepsilon_{1}}\right),
$$

where $\varepsilon_{1}=\frac{r_{k}^{n_{1}+n_{2}}}{8 B\left(H_{\delta_{k}, r_{k}}\left(P_{\eta_{k}}\right) B+\left(1-r_{k}\right)^{-1}\right)}$.

Proof. We have

$$
\left\||| P_{\eta_{k}}-P_{\eta}\right\||\leq||| P_{\eta_{k}}-P\left\||+||| P-P_{\eta}\right\| \mid \leq 2 \varepsilon_{k} \Gamma \leq \varepsilon_{0}\left(P_{\eta_{k}}, r_{k}, \delta_{k}\right) .
$$

Therefore, we can use Theorem 2.2 with $P_{\eta_{k}}=P_{1}$ and $P_{\eta}=P_{2}$. Thus, we obtain

$$
\begin{aligned}
\left\|\left(z-P_{\eta}\right)^{-1} f\right\|_{B V} & \leq \frac{4(A+B)}{1-r_{k}} r_{k}^{n_{1}}\|f\|_{B V}+\frac{1}{2 \varepsilon_{1}}\|f\|_{1} \\
& \leq\left(\frac{4(A+B)}{1-r_{k}} r_{k}^{n_{1}}+\frac{1}{2 \varepsilon_{1}}\right)\|f\|_{B V} .
\end{aligned}
$$

Remark 4.3. We are now in a situation where $\left|\rho_{\eta_{k}}-\rho_{\eta}\right|<\delta_{k},\left|\rho_{\eta_{k}}-\rho\right|<\delta_{k}$ and $\left|\rho_{\eta}-\rho\right|<\delta_{k}$ and $\rho$ is at a distance of at least $2 \delta_{k}$ from any non dominant eigenvalue of $P, P_{\eta_{k}}$ and $P_{\eta}$. 
Now, we can define the spectral projection [8] associated with $\rho_{\eta}$ by

$$
\Pi_{\rho_{\eta}}=\frac{1}{2 \pi i} \int_{\partial B\left(\rho, 2 \delta_{k}\right)}\left(z-P_{\eta}\right)^{-1} d z .
$$

Lemma 4.4. Let $\varepsilon=\operatorname{mesh}(\eta)<\varepsilon_{k}$. We have

$$
|| \Pi_{\rho_{\eta}}-\Pi_{\rho} \| \mid \leq C_{0} \varepsilon^{\gamma},
$$

where

$$
C_{0}=2 \delta_{k} \Gamma^{\gamma}\left(a\left(\frac{4(A+B)}{1-r_{k}} r_{k}^{n_{1}}+\frac{1}{2 \varepsilon_{1}^{*}}\right)+b\left(\frac{4(A+B)}{1-r_{k}} r_{k}^{n_{1}}+\frac{1}{2 \varepsilon_{1}^{*}}\right)^{2}\right),
$$

and

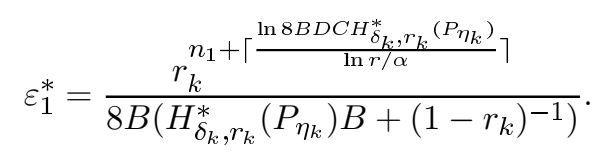

Proof. Theorem 2.2 implies

$$
\begin{aligned}
\left\|\Pi_{\rho_{\eta}}-\Pi_{\rho} \mid\right\| & \leq \frac{1}{2 \pi} \int_{\left\{z \in \mathbb{C}:|z-\rho|=2 \delta_{k}\right\}} \|\left(z-P_{\eta}\right)^{-1}-(z-P)^{-1}|||| d z \mid \\
& \leq \sup _{\left\{z \in \mathbb{C}:|z-\rho|=2 \delta_{k}\right\}}\left(a\left\|\left(z-P_{\eta}\right)^{-1}\right\|_{B V}+b\left(\left\|\left(z-P_{\eta}\right)^{-1}\right\|_{B V}\right)^{2}\right) \cdot 2 \delta_{k} \Gamma^{\gamma} \varepsilon^{\gamma} .
\end{aligned}
$$

Since $z \in \mathbb{C} \backslash V_{\delta_{k}, r_{k}}\left(P_{\eta_{k}}\right)$ and $\varepsilon_{1} \geq \varepsilon_{1}^{*}$, Lemma 4.2 implies

$$
\sup _{\left\{z \in \mathbb{C}:|z-\rho|=2 \delta_{k}\right\}}\left\|\left(z-P_{\eta}\right)^{-1}\right\|_{B V} \leq \frac{4(A+B)}{1-r_{k}} r_{k}^{n_{1}}+\frac{1}{2 \varepsilon_{1}^{*}} .
$$

This completes the proof.

Remark 4.5. The constant $C_{0}$ in Lemma 4.4 is computable.

Theorem 4.6. Let $\varepsilon=m e s h(\eta)<\varepsilon_{k}$. Let $g$ and $g_{\eta}$ be the normalized eigenvectors associated with $\rho$ and $\rho_{\eta}$ respectively. Then:

(1) $\left\|g-g_{\eta}\right\|_{1} \leq \frac{2 \bar{C}}{1-\bar{C} \varepsilon^{\gamma}} \varepsilon^{\gamma}$,

(2) $\left|\rho-\rho_{\eta}\right| \leq \frac{2 \bar{C}}{1-\bar{C} \varepsilon^{\gamma}} \varepsilon^{\gamma}$,

where $\bar{C}=C_{0}\left(1+\frac{B_{0}}{r_{k}-\alpha}\right)$.

Proof. Let $g$ be the normalized eigenvector corresponding to $\rho$; i.e., $g \geq 0$ and $\|g\|_{1}=1$. Define $\bar{g}_{\eta} \stackrel{\text { def }}{=} \Pi_{\rho_{\eta}} g$. Firstly, we estimate the $B V$ norm of $g$. We have, by (A1),

$$
\rho(V g)=V P g \leq \alpha V g+B_{0} .
$$

Therefore,

$$
V g \leq \frac{B_{0}}{r_{k}-\alpha}
$$

and

$$
\|g\|_{B V} \leq \frac{B_{0}}{r_{k}-\alpha}+1
$$


We now use this and Lemma 4.4 to get an estimate on the $L^{1}$ norm of $\bar{g}_{\eta}$. We have

$$
\begin{aligned}
\|g\|_{1}-\left\|\bar{g}_{\eta}\right\|_{1} & \leq\left\|g-\bar{g}_{\eta}\right\|_{1} \\
& =\left\|\Pi_{\rho} g-\Pi_{\rho_{\eta}} g\right\|_{1} \\
& \leq\|\| \Pi_{\rho}-\Pi_{\rho_{\eta}}\|\| \cdot\|g\|_{B V} \\
& \leq C_{0} \varepsilon^{\gamma}\left(\frac{B_{0}}{r_{k}-\alpha}+1\right)=\bar{C} \varepsilon^{\gamma} .
\end{aligned}
$$

Thus, $\left\|\bar{g}_{\eta}\right\|_{1} \in\left(1-\bar{C} \varepsilon^{\gamma}, 1+\bar{C} \varepsilon^{\gamma}\right)$. Let $g_{\eta} \stackrel{\text { def }}{=} \frac{\bar{g}_{\eta}}{\left\|\bar{g}_{\eta}\right\|_{1}}$. Then

$$
\begin{aligned}
\left\|g_{\eta}-g\right\|_{1} & \leq\left\|\frac{\bar{g}_{\eta}}{\left\|\bar{g}_{\eta}\right\|_{1}}-\frac{g}{\left\|\bar{g}_{\eta}\right\|_{1}}\right\|_{1}+\left\|\frac{g}{\left\|\bar{g}_{\eta}\right\|_{1}}-g\right\|_{1} \\
& \leq \frac{1}{\left\|\bar{g}_{\eta}\right\|_{1}}\left\|\bar{g}_{\eta}-g\right\|_{1}+\frac{\left|1-\left\|\bar{g}_{\eta}\right\|_{1}\right|}{\left\|\bar{g}_{\eta}\right\|_{1}}\|g\|_{1} \\
& \leq \frac{\bar{C} \varepsilon^{\gamma}}{1-\bar{C} \varepsilon^{\gamma}}+\frac{\bar{C} \varepsilon^{\gamma}}{1-\bar{C} \varepsilon^{\gamma}}=\frac{2 \bar{C}}{1-\bar{C} \varepsilon^{\gamma}} \varepsilon^{\gamma} .
\end{aligned}
$$

Finally, we can obtain an estimate on the distance between $\rho$ and $\rho_{\eta}$ :

$$
\begin{aligned}
\left|\rho_{\eta}-\rho\right| & =\left|\left\|P_{\eta} g_{\eta}\right\|_{1}-\|P g\|_{1}\right| \\
& =\|\|\left(\Pi_{\eta} \circ P \circ \Pi_{\eta}\right) g_{\eta}\left\|_{1}-\right\| P g\left\|_{1}|=|\right\| P g_{\eta}\left\|_{1}-\right\| P g \|_{1} \mid \\
& \leq\left\|P g_{\eta}-P g\right\|_{1} \leq\left\|g_{\eta}-g\right\|_{1} \leq \frac{2 \bar{C}}{1-\bar{C} \varepsilon^{\gamma}} \varepsilon^{\gamma} .
\end{aligned}
$$

\section{EXAMPLE}

In this section we use Algorithm I to approximate the escape rate of a LasotaYorke map with holes. We analyse a simple example to demonstrate the performance and feasiblity of the algorithm.

Example 5.1. We consider the transformation

$$
T(x)=\left\{\begin{array}{cl}
2.08 x & \text { for } x<\frac{1}{2} \\
2-2 x & \text { for } x \geq \frac{1}{2}
\end{array} .\right.
$$

Remark 5.2. We chose a piecewise linear example so that the escape rate may be computed directly, and thereafter compared to the estimate derived from the algorithm. In fact, for $U \in \mathfrak{B}(I)$, we have

$$
\lambda\left(T^{-1}(U)\right)=\frac{1}{2.08} \lambda(U)+\frac{1}{2} \lambda(U)=\frac{4.08}{4.16} \lambda(U) .
$$

Thus, the exact dominant eigenvalue of $P$ is $\rho=\frac{4.08}{4.16}$.

\section{Calculations for the algorithm}

First we will show that the Perron-Frobenius operator $P$ associated with $T$ satisfies (A1) and (A2). It is easily seen that for $f \in B V(I)$ we have

$$
V P f \leq \frac{1}{2} V f .
$$


Thus, (A1) is satisfied with $\alpha=\frac{1}{2}$ and $B_{0}=0$. Consequently,

$$
\|P f\|_{B V} \leq \frac{1}{2} V f+\|f\|_{1} .
$$

We now check that (A2) is satisfied without using Remark 5.2. Thus, the following is a procedure for checking that (A2) is satisfied without knowing $\rho$ a priori.

Let $H=\left(\frac{1}{2.08}, \frac{1}{2}\right)$. Then $\lambda(H)=\frac{0.08}{4.16}$, where $H$ is the hole in $[0,1]$ from which the particles escape under the evolution of $T$. Let $q \in\left(0, \frac{1 / 2-\lambda(H)}{\lambda(H)}\right)$ and define

$$
E_{q}=\left\{f \in L^{1}: V f<q, f \geq 0, \int_{I} f d \lambda=1\right\}
$$

Then $E_{q}$ is a compact convex subset of $L^{1}(I)$. For $f \in E_{q}$

$$
\begin{aligned}
\|P f\|_{1}=1-\int_{H} f d \lambda & \geq 1-\lambda(H) \cdot\|f\|_{\infty} \\
& \geq 1-\lambda(H)(V f+1)>\frac{1}{2}=\alpha .
\end{aligned}
$$

Let

Then, for $f \in E_{q}$,

$$
\bar{P} f=\frac{P f}{\|P f\|_{1}} .
$$

$$
V(\bar{P} f) \leq\|P f\|_{1}^{-1} V(P f)<V f .
$$

Thus $\bar{P}\left(E_{q}\right) \subset E_{q}$. The Schauder-Tychonoff Theorem [5] implies $\bar{P}$ has a fixed point in $E_{q}$; i.e.,

Or

$$
\frac{P g}{\|P g\|_{1}}=g
$$

$$
P g=\rho g,
$$

where $\rho=\|P g\|_{1}>\alpha$. Thus, (A2) is satisfied.

Now, we implement Algorithm I and obtain rigorous approximation of $\rho$. From inequality (5.1), we obtain that $\alpha=\frac{1}{2}$ and $B_{0}=0$. Let the tolerance on the approximation error be $\tau=0.01$. Let $k=50$ and pick $\delta=\frac{1}{2 k}$ and $r=\alpha+\frac{23.5}{k}$. Notice that we have modified $r$ from $\alpha+\frac{1}{k}$ to $\alpha+\frac{23.5}{k}$. Since the algorithm works for any decreasing sequences of $r$ 's and $\delta$ 's, this modification does not affect the proofs of the previous sections. We picked up this sequence of $r$ 's because it will require the outer loop of the algorithm to run only once. Table 1 shows the output of the algorithm after running the inner loop twice. In the first run, we took the mesh size $\varepsilon=2 \times 10^{-4}$ which failed to satisfy $\varepsilon<(2 \Gamma)^{-1} \varepsilon_{0}^{*}$. Then, we reduced the mesh size to $\varepsilon=1.25 \times 10^{-4}$ and it satisfied $\varepsilon<(2 \Gamma)^{-1} \varepsilon_{0}^{*}$. Thus, we obtained a rigorous approximation of $\rho$. We have used MATLAB in our computations. The main difficulty in implementing the algorithm is the computation of $H_{\delta, r}^{*}$. In the discussion below, we will explain how we have computed $H_{\delta, r}^{*}$ for this particular example.

We present the computation of $H_{\delta, r}^{*}$ when $\varepsilon=\operatorname{mesh}(\eta)=1.25 \times 10^{-4}$. The computation of $H_{\delta, r}^{*}$ when $\varepsilon=\operatorname{mesh}(\eta)=2 \times 10^{-4}$ is done in exactly the same way. 


\begin{tabular}{|c|c|c|}
\hline$\delta$ & $10^{-2}$ & $10^{-2}$ \\
\hline$r$ & 0.97 & 0.97 \\
\hline$\varepsilon$ & $2 \times 10^{-4}$ & $1.25 \times 10^{-4}$ \\
\hline$\rho_{\eta}$ & 0.98076929201212 & 0.98076931645293 \\
\hline$H_{\delta, r}^{*}$ & 162.9499805 & 165.1996625 \\
\hline$(2 \Gamma)^{-1} \varepsilon_{0}^{*}$ & $1.30392654 \times 10^{-4}$ & $1.289151061 \times 10^{-4}$ \\
\hline Inner Loop & Reduce Mesh Size & Pass \\
\hline Outer Loop & Use Same $\delta$ and $r$ & Pass \\
\hline Report & No Report & $|\rho-0.98076931645293|<10^{-2}$ \\
\hline \multicolumn{3}{|c|}{ TABLE 1} \\
\hline
\end{tabular}

After choosing $\varepsilon=\operatorname{mesh}(\eta)=1.25 \times 10^{-4}$ and setting the matrix $P_{\eta}$, we find its dominant eigenvalue $\rho_{\eta}$ and its second largest eigenvalue. We noticed that $\rho_{\eta}$ is simple and that the modulus of second largest eigenvalue is smaller than $\alpha$. Thus, $\sigma\left(P_{\eta}\right) \backslash\left\{\rho_{\eta}\right\} \subset B(0, \alpha)$. We have (see [8])

$$
\left\|\left(z-P_{\eta}\right)^{-1}\right\|_{1} \leq \delta^{-1}\left\|\Pi_{\rho_{\eta}}\right\|_{1}+\|R(z)\|_{1},
$$

where $\left\|\Pi_{\rho_{\eta}}\right\|$ is the rank-one projection associated with $\rho_{\eta}$ and $R(z)$ is the resolvent of the operator $P_{\eta}\left(\mathbf{1}-\Pi_{\rho_{\eta}}\right)$. Since the modulus of the second largest eigenvalue of $P_{\eta}$ is smaller than $\alpha$ and $|z|>r>\alpha, R(z)$ can be represented by a convergent Neumann series. We have

$$
\begin{aligned}
\|R(z)\|_{1} & \left.=\| \sum_{n=0}^{\infty} \frac{\left(P_{\eta}\left(\mathbf{1}-\Pi_{\rho_{\eta}}\right)\right)^{n}}{z^{n+1}}\right) \|_{1} \\
& \leq \frac{1}{r}\left(\sum_{n=0}^{17} \frac{\left\|\left(P_{\eta}\left(\mathbf{1}-\Pi_{\rho_{\eta}}\right)\right)^{n}\right\|_{1}}{r^{n}}+\sum_{n=18}^{\infty} \frac{\left\|\left(P_{\eta}\left(\mathbf{1}-\Pi_{\rho_{\eta}}\right)\right)^{n}\right\|_{1}}{r^{n}}\right) \\
& \leq \frac{1}{r}\left[\sum_{n=0}^{17} \frac{\left\|\left(P_{\eta}\left(\mathbf{1}-\Pi_{\rho_{\eta}}\right)\right)^{n}\right\|_{1}}{r^{n}}\left(1+\sum_{m=1}^{\infty}\left(\frac{\left\|\left(P_{\eta}\left(\mathbf{1}-\Pi_{\rho_{\eta}}\right)\right)^{18}\right\|_{1}}{r^{18}}\right)^{m}\right)\right] \\
& <34.27921938 .
\end{aligned}
$$

We have used the fact that $\left\|\left(P_{\eta}\left(\mathbf{1}-\Pi_{\rho_{\eta}}\right)\right)^{18}\right\|_{1}<0.04169901783$, to obtain a convergent geometric series $\sum_{m=1}^{\infty}\left(\frac{\left\|\left(P_{\eta}\left(\mathbf{1}-\Pi_{\rho_{\eta}}\right)\right)^{18}\right\|_{1}}{r^{18}}\right)^{m}$. Computing the $L^{1}$ norm of the projection

$$
\left\|\Pi_{\rho_{\eta}}\right\|_{1}=1.257
$$

and using the definition of $H_{\delta, r}^{*}$ and inequality (5.3), we obtain that

$$
H_{\delta, r}^{*} \leq 165.1996625 \text {. }
$$

Therefore,

$$
(2 \Gamma)^{-1} \varepsilon_{0}^{*} \geq 1.289151061 \times 10^{-4}>1.25 \times 10^{-4}=\varepsilon .
$$

Thus, the inner loop of the algorithm is satisfied. The outer loop is also satisfied since $\bar{B}(0, r) \cap \bar{B}\left(\rho_{\eta}, \delta\right)=\emptyset$. Thus, $0.9707<\rho<0.9908$.

Remark 5.3. The interval $(0.9707,0.9908)$ does indeed contain the exact dominant eigenvalue of $P$ which was calculated in Remark 5.2. 


\section{Remarks on estimating $H_{\delta, r}^{*}$}

(1) It is clear that the main numerical difficulty in implementing the algorithm is the rigorous estimation (from above) of the quantity $H_{\delta, r}^{*}$. The calculation in the example above shows one way of doing this whenever $P_{\eta}$ has a single, simple dominant eigenvalue outside of the disc $B(0, \alpha)$.

(2) In cases when the spectrum of $P_{\eta}$ (and hence $P$ ) is more complicated on $(B(0, \alpha))^{c}$, the rigorous estimation of $H_{\delta, r}^{*}$ is an interesting and challenging problem.

Acknowledgment: The revised version of the paper has benefited from enlightening suggestions of anonymous referees and the editorial board member. It is my pleasure to thank Professor Christopher Bose for his encouragement, support, helpful discussions and his criticism for an older version of the paper. I would like to thank Professor Rua Murray for helpful discussions during his visits to the Department of Mathematics and Statistics at the University of Victoria. I would also like to thank Professor Boualem Khouider for giving me access to the Research Computing Facility at the University of Victoria, and John Foxgord for his technical support.

\section{REFERENCES}

1. Baladi, V., Positive transfer operators and decay of correlations, Advanced Series in Nonlinear Dynamics, 16. World Sci. Publ., NJ, 2000.

2. van den Bedem, H. and Chernov, N., Expanding maps of an interval with holes, Ergod. Th. Dynam. Sys. 22 (2002), no. 3, 637-654.

3. Collet, P., Martínez, S. and Schmitt, B., On the enhancement of diffusion by chaos, escape rates and stochastic instability, Trans. Amer. Math. Soc. 351 (1999), no. 7, 2875-2897.

4. Demers, M. and Young, L-S., Escape rates and conditionally invariant measures, Nonlinearity 19 (2006), 377-397.

5. Dunford, N., and Schwartz, J.T., Linear Operators, Part I: General Theory, Interscience Publ. Inc., N.Y., 1964.

6. Gaspard, P., and Dorfman, R., Chaotic scattering theory, thermodynamic formalism, and transport coefficients, Phys. Rev. E 52 (1995), 3525-3552.

7. Gaspard, P., Dynamical chaos and nonequilibrium statistical mechanics, Internat. J. Modern Phys. B 15 (2001), no. 3, 209-235.

8. Kato, T., Perturbation theory for linear operators, Springer-Verlag, NY, 1966.

9. Keller, G. and Liverani, C., Stability of the spectrum for transfer operators, Ann. Scuola Norm. Sup. Pisa Cl. Sci. (4) 28 (1999), no. 1, 141-152.

10. Lancaster, P. and Tismenetsky, M., The theory of matrices, Academic Press, Inc., Orlando, 1985.

11. Liverani, C. Rigorous numerical investigation of the statistical properties of piecewise expanding maps. A feasibility study, Nonlinearity, 14 (2001), no. 3, 463-490.

12. Liverani, C. and Maume-Deschamps, V., Lasota-Yorke maps with holes: conditionally invariant probability measures and invariant probability measures on the survivor set, Ann. Inst. H. Poinc. Probab. Statist. 39 (2003), no. 3, 385-412.

13. Murray, R., Discrete approximation of invariant densities, PhD Thesis University of Cambridge, 1997.

14. Pianigiani, G. and Yorke, J., Expanding maps on sets which are almost invariant. Decay and chaos, Trans. Amer. Math. Soc. 252 (1979), 351-366.

15. Ruelle, D., Entropy production in nonequilibrium statistical mechanics, Commun. Math. Phys., (1997), 189, 365-371.

16. Ulam, S.M., A Collection of Mathematical Problems, Interscience, New York, Interscience Tracts in Pure and Applied Math., 8, 1960.

17. Wilkinson, J. H., The algebraic eigenvalue problem, Clarendon Press, Oxford, 1965. 
Department of Mathematics and Statistics, University of Victoria, PO BOX 3045 STN CSC, ViCTORIA, B.C., V8W 3P4, CANAdA

E-mail address: wab@math.uvic.ca 